\title{
Aplicación de las Tecnologías de la Información y la Comunicación (TIC) en los Procesos de Enseñanza- Aprendizaje por parte de los Profesores de Química
}

\author{
Luz D. Martínez-Argüello, Francisco J. Hinojo-Lucena e Inmaculada Aznar Díaz* \\ Univ. de Granada, Fac. de Ciencias de la Educación, Dpto de Didáctica y Organización Escolar, Campus \\ Universitario de Cartuja, C.P.18071, Granada- España. (e-mail: luzdary455@hotmail.com; fhinojo@ugr.es; \\ iaznar@ugr.es)
}

${ }^{*}$ Autor a quien debe ser enviada la correspondencia

Recibido Jul. 17, 2017; Aceptado Sep. 26, 2017; Versión final Nov. 22, 2017, Publicado Abr. 2018

\begin{abstract}
Resumen
Se presenta un estudio sobre cómo los profesores de química de seis instituciones públicas de Bucaramanga-Colombia se integran a la nueva cultura digital en sus clases. Para tal propósito se aplicaron cuestionarios a 210 estudiantes y seis profesores de química. Además se realizaron entrevistas estructuradas a los maestros. El estudio reveló que los videos y artículos, seguido del desarrollo de clases usando PowerPoint son las formas de uso más frecuente de las Tecnologías de la Información y la Comunicación (TIC) en la enseñanza-aprendizaje de la química. Las temáticas en las que se han incorporado han sido: propiedades de gases, propiedades de la materia, reacciones químicas, enlaces, polímeros, petróleo, caucho y átomo de carbono. Los maestros manifestaron que nunca habían planeado clases que involucraran el uso de programas, tutoriales o simuladores para química. Los resultados permiten concluir que hay un limitado uso de las TIC en la enseñanza de la química.
\end{abstract}

Palabras claves: TIC; enseñanza-aprendizaje de la química; didáctica de las ciencias; alfabetización tecnológica; cultura digital

\section{Application of Information and Communication Technologies (ITC) in Teaching-Learning Processes by Chemistry Teachers}

\begin{abstract}
A study on how chemistry teachers of six public institutions in Bucaramanga-Colombia incorporate Information and Communication Technologies (ITC) in the teaching-learning processes in their lectures is presented. Questionnaires were applied to six chemistry professors and 210 students between 15 and 20 years of age. In addition, structured interviews were conducted with teachers. The study revealed that the videos and articles, followed by the development of classes with the use of Power Point, are the ways in which ICT are often incorporated into the teaching-learning chemistry processes. The most common topics for using ICT are properties of gases, properties of materials, chemical reactions, bonds, polymers, petroleum, rubber and the carbon atom. Teachers expressed that they never planned a class involving the use of software programs, tutorials or simulators in chemistry. The results show that there is limited use of ICT in the teaching chemistry.
\end{abstract}

Keywords: TIC; teaching- learning of chemistry; didactics of science; technological literacy; digital culture 


\section{INTRODUCCIÓN}

Colombia es considerada pionera a nivel mundial, en la implementación de Tecnologías de la Información y Comunicación (TIC) con fines educativos: Radio Sutatenza desde su fundación en 1947, cubrió buena parte del territorio nacional, con el objetivo de llegar a la población rural, caracterizada, entre otras cosas, por no tener acceso a la educación (Bernal, 2005). Hoy día, en el marco de la política nacional y el Plan Nacional de Desarrollo 2014-2018 "Todos por un nuevo país", se trazó como objetivo hacer de Colombia el país mejor educado de América Latina en el año 2025, donde el uso y aplicación de las TIC dentro de las aulas estaría direccionado a contribuir a alcanzar dicho objetivo. Entre otras cosas porque mediante el uso de TIC en la educación se contribuye a reducir la brecha digital existente, se brindan más y mejores oportunidades a todos los sectores sociales, especialmente a los más vulnerables y de esa manera se convierte en un factor de calidad de vida, disminución de pobreza y equidad social (Presidencia de la República, 2014).

Con este propósito, el gobierno ha venido realizando ajustes y llevando a cabo políticas que permitieran preparar a la nación para este gran reto. Es así como en el 2001 nació el programa "Computadores para educar", una iniciativa de la Presidencia de la República que goza con la ayuda mancomunada del sector público y privado, cuyo objetivo ha sido que todos los niños y jóvenes del país tengan acceso a las nuevas tecnologías de la información y la comunicación (Computadores para educar, 2011). Para el 2011 se incorporaron al programa contenidos digitales avalados por el Ministerio de Educación Nacional (MEN) con el propósito de orientar las áreas del conocimiento a la luz de los estándares y contribuir al desarrollo de competencias en los educandos. Dentro de los contenidos se encuentran tutoriales para diferentes áreas del conocimiento y bases de datos científicas (MINTIC, 2012) y el programa fue elegido como "proyecto que genera aprendizajes que pueden ser replicados en otros lugares del mundo" (UNESCO, 2012).

En mayo del 2012 además se anunció un programa que tuvo como objetivo la formación de maestros en un plan que comprendía 150 horas presenciales en cada sede educativa con el fin de incorporar las TIC en las aulas. Desde entonces los esfuerzos que se han hecho por evitar quedar al margen de la sociedad del conocimiento y de sus herramientas han sido continuos, de tal manera que la formación de los maestros y la adecuación y dotación de herramientas TIC son una prioridad dentro de la política nacional. El objetivo de hacer de Colombia el país más educado de América Latina presenta, entre otros retos, mejorar los niveles de éxito tanto en pruebas nacionales como internacionales, donde ha quedado relegada en comparación con otros países de la región (OCDE, 2016). Las pruebas internacionales se han caracterizado por medir el nivel de competencias de los educandos en ciencias, matemáticas y lectura. En el caso particular de las ciencias, hay mucho por hacer, pues los resultados obtenidos en esta materia no han sido alentadores. Así mismo, la falta de interés y rechazo hacia el estudio de las ciencias (Cañal, 2012) es motivo de preocupación, debido entre otras cosas, a prácticas educativas inadecuadas. Por tanto se deben diseñar estrategias pedagógicas conducentes a subsanar esta situación y sean parte de la construcción de la Colombia educada, próspera, equitativa y en paz que se desea tener.

En este contexto educativo las TIC pueden ser un elemento que favorezca los procesos de enseñanzaaprendizaje, porque inciden directamente en la motivación de los estudiantes que se ven atraídos por ellas, favorecen aprendizajes flexibles en cuanto al rol del profesor y el alumno y potencializan escenarios interactivos (Cabero, 2007). Concretamente para la enseñanza de la química, Orlik (2002) hace una descripción amplia de los beneficios que las TIC proporcionan, destacando entre otros, software, simulaciones, laboratorios, bibliotecas virtuales, videoconferencias, tutorías, foros, herramientas para la evaluación y el refuerzo. Igualmente otros autores han ofrecido diferentes alternativas para obtener el máximo aprovechamiento de las TIC mediante su correcta utilización en los procesos formativos (Barroso y Llorente 2007).

La enseñanza a través de las TIC como herramienta o instrumento de los procesos de enseñanza aprendizaje ha dado origen a lo que se ha denominado: las tecnologías del aprendizaje y el conocimiento o TAC (Espuny, et al., 2010), donde se busca generar el aprendizaje con la tecnología. Según Sancho Gil (2008) la transformación de TIC a TAC sólo es posible si se genera un cambio en la práctica docente y se le da un valor aparte del motivador, como lo es la retroalimentación y con él y la práctica educativa se está generando los tan anhelados aprendizajes significativos: "Los medios no funcionan en un contexto vacío, sino que más bien lo hacen en un contexto complejo e incierto, donde los resultados y la eficacia que se consiga con ellos dependerá de la interacción de un número de variables que van desde los materiales e instrumentales hasta las personales y organizativas" Cabero (1997). La incorporación de las TIC dentro de las prácticas educativas contribuye a la ampliación de la cultura digital mediante la alfabetización tecnológica que se le proporciona a los educandos. En opinión de Salcedo (2008) con la incorporación de las TIC a la enseñanza de la química se proporciona un entorno familiar entre el estudiante y la forma como se encuentra conectada la química, la tecnología y la sociedad de la información. Además se propicia la formación de ciudadanos alfabetizados científicamente. 
Sin embargo, hay un buen número de docentes con una posición poco favorable hacia las TIC (Hinojo, 2002), que opinan que para poder implementar el uso de las TIC se requiere destinar una mayor cantidad de tiempo en la preparación de las clases, aparte del esfuerzo adicional que exigen su uso y manejo. Con estas manifestaciones se evidencia un problema didáctico y de metodología que genera un conflicto en el docente al no identificar cómo y cuándo hacer uso de una determinada TIC, privándose él y sus estudiantes del mundo versátil y motivador que cada una de ellas proporcionan además de someterlos a la exclusión dentro del mundo globalizado donde las TIC ocupan un lugar preponderante.

En opinión de Talanquer (2004), para ser un buen profesor de química no son suficientes los sólidos conocimientos en el área, hacen falta otros componentes igualmente importantes que no sólo facilitan el proceso de enseñanza- aprendizaje sino que además contribuyen a la corresponsabilidad de educar jóvenes en competencias científicas. El conocimiento disciplinar, pedagógico (Shulman, 1986), y otros de carácter algo más intangible pero que caracterizan a cada profesor sobre la forma de cómo lleva a cabo sus procesos de enseñanza- aprendizaje, se ha denominado conocimiento pedagógico del contenido (CPC). En otras palabras el conocimiento conceptual y de base de una materia, sumado a las estrategias de enseñanza conforman el CPC. Si aunado a lo anterior se emplean prácticas docentes que incluyan el uso de tecnologías, se estarían formando jóvenes no sólo alfabetizados científicamente (Martínez y Quijano, 2010), sino que además estarían alfabetizados tecnológica y digitalmente.

Tanto Shulman como los posteriores autores del (CPC) no tienen en cuenta como elemento destacado del profesional docente el uso de las TIC. Es tan sólo a partir del siglo XXI cuando se comprende, que para que las TIC se conviertan en elementos de enseñanza-aprendizaje dentro de los centros educativos, es necesario que los docentes no sólo posean un CPC sino que además necesitan un conocimiento sobre qué TIC son convenientes y adecuadas para el objeto de su enseñanza (Marcelo, et al., 2016). Este conocimiento integrado se ha denominado conocimiento tecnopedagógico del contenido (TPACK o TPCK) (Niess, 2005, Hechter, Phyfe y Vermette 2012). Podemos asumir por tanto que el nivel de uso de las TIC en sus procesos de enseñanza-aprendizaje son un indicativo del conocimiento que poseen sobre ellas para su implementación (Albion et al., 2010). En ese sentido, para esta investigación se ha planteado: ¿qué aplicación le dan los profesores de la media vocacional de química a las TIC en sus procesos de enseñanza- aprendizaje?

\section{METODOLOGÍA}

En este trabajo se desarrolló una metodología ecléctica o mixta, caracterizada por considerar tanto el aspecto cualitativo como cuantitativo. El alcance del aspecto que corresponde a la investigación cuantitativa es descriptivo e inferencial. Las técnicas utilizadas en este trabajo han sido la encuesta y la entrevista estructurada. Las encuestas se aplicaron a profesores de química y estudiantes de los dos últimos grados de enseñanza media.

Por otro lado, también se realizaron entrevistas estructuradas a los docentes. Tanto en la aplicación de encuestas como en la entrevista estructurada se aplicaron cuestionarios previamente diseñados con el fin de recoger información de forma conjunta sobre los conceptos y las variables de interés (Hernández, et al., 2010).

Salvo algunas pequeñas diferencias, los cuestionarios tanto para los estudiantes, como para los docentes, están organizados en cuatro dimensiones: Información personal, Instalaciones y recursos físicos, Concepciones sobre la utilización de las TIC e Incorporación de las TIC en la enseñanza de la química. El número de ítems para cada dimensión ha sido de 8, 14,11, 20 respectivamente.

Para cada ítem se asignaron cuatro alternativas de respuestas tipo Likert de cuatro puntos para escoger la que expresara mejor su grado de conformidad o frecuencia frente a ellos. De conformidad: 1: Totalmente en desacuerdo, 2: Poco de acuerdo, 3: De acuerdo; 4: Totalmente de acuerdo. De frecuencia: 1: Nunca, 2: Algunas veces, 3: Frecuentemente, 4: Siempre.

Dado que se trata de un estudio cuasi-experimental en contextos naturales y ecológicos, como son las instituciones educativas, se ha realizado un muestreo no probabilístico, deliberado, basado en la organización de los centros escolares contemplados en esta investigación y cuyas aulas de clase ya estaban predeterminadas desde el comienzo del año escolar (Hernández, et al., 2010; Morales, 2013).

La población total de estudiantes fue de 728. Para encontrar el tamaño de la muestra se utilizó la fórmula determinada para su cálculo en poblaciones finitas, cuya expresión matemática se muetra en la ecn. (1). El significado de los parámetros y variables de la ecn. (1) se muestra en la Tabla 1. 


$$
n=\frac{Z^{2} a * N * p * q}{i^{2}(N-1)+Z^{2} a * p * q}
$$

Tabla 1: Parámetros de la fórmula usada para determinar poblaciones finitas

\begin{tabular}{|c|l|c|}
\hline Parámetro & \multicolumn{1}{|c|}{ Descripción del parámetro } & Valor \\
\hline$N$ & Tamaño de la muestra & 728 \\
\hline$N$ & Tamaño de la población & 1,96 \\
\hline$Z_{\alpha}$ & $\begin{array}{l}\text { Valor correspondiente al área de la curva de distribución normal para un valor de } \\
\text { alfa = 0,05 (cuando el nivel de confianza es de 95\%) }\end{array}$ & 0,5 \\
\hline$p$ & $\begin{array}{l}\text { Máxima probabilidad esperada del parámetro a evaluar, al desconocerse se utiliza } \\
p=50 \% \text { que hace mayor el tamaño muestral }\end{array}$ & 0,5 \\
\hline$q$ & Complemento de p, q = (1 - p) & 0,06 \\
\hline$i$ & $\begin{array}{l}\text { Precisión. Según la finalidad de este estudio, se requiere una precisión media que } \\
\text { se estima en el 6\% }\end{array}$ &
\end{tabular}

Reemplazando en la expresión (1) los valores correspondientes de la Tabla 1, se obtiene un valor para n=196, es decir, esta es la cantidad mínima de estudiantes que se necesitan para que la muestra sea válida estadísticamente. Buscando la participación equitativa de todos los grupos de clase, se decidió escoger aleatoriamente 8 alumnos de cada una de las aulas (un total de 26) para aplicarles el cuestionario.

En la investigación han participado seis docentes de química, cinco de ellos en edades alrededor de los cuarenta y uno mayor de sesenta años. Por el grupo de estudiantes, 210 alumnos de los grados décimos y undécimos de enseñanza media (14-20 años) de seis Institutos Públicos de Educación Secundaria del núcleo 3 de la ciudad de Bucaramanga-Colombia. Estos estudiantes han cursado o cursan la materia de química, sin adaptación curricular. La aplicación del cuestionario se ha realizado al final de las unidades didácticas, con aviso previo y después de impartir los contenidos curriculares correspondientes a ocho estudiantes escogidos al azar por cada grupo de clase en estos niveles.

Los datos obtenidos con la aplicación del cuestionario se sometieron a digitalización en el software Microsoft ${ }^{\circledR}$ Excel 2010. La base de datos validada se analizó a través del software estadístico Statistical Package for the Social Sciences ${ }^{\circledR}$ (SPSS 23.0) y Système Portable pour l'Analyse de Données $₫$ (SPAD V56).

El alfa de Cronbach tiene gran utilidad para determinar la consistencia interna de una prueba con una dimensión, y para no correr el riesgo de subestimarla cuando se tienen varias dimensiones, Oviedo y Campo-Arias (2005) recomienda calcular su valor para cada uno de los grupos de ítems que componen una dimensión o una subescala. Al mirar dicho valor del coeficiente para toda la escala es igual a 0,893 (considerado como bueno) se puede determinar que la fiabilidad del instrumento apoya la interpretación de que el cuestionario se puede considerar fiable (Tabla 2).

Tabla 2: Resultados del análisis de fiabilidad de la encuesta a los estudiantes

\begin{tabular}{|l|l|l|}
\hline \multicolumn{2}{|c|}{ Coeficiente alfa de Cronbach } \\
\hline \multirow{4}{*}{ Dimensiones } & Instalaciones y recursos físicos & 0,721 \\
\cline { 2 - 3 } & Concepciones sobre el manejo de las TIC & 0,480 \\
\cline { 2 - 3 } & Incorporación de las TIC a la enseñanza de la química & 0,880 \\
\cline { 2 - 3 } & Incorporación de las TIC en la clase de química & 0,887 \\
\hline Toda la escala & 0,893 \\
\hline
\end{tabular}

\section{RESULTADOS}

Se analizaron las respuestas dadas por los docentes en sus entrevistas y cuestionarios y se contrastaron estos con las respuestas dadas por los estudiantes participantes. Los resultados mostraron una gran coherencia entre las percepciones manifestadas tanto en maestros como en estudiantes.

\section{Las TIC facilitan el aprendizaje de la química}

El $82,2 \%$ de los estudiantes encuestados manifestaron que con la utilización de las TIC el aprendizaje de la química se vería facilitado (figura 1); igual opinión tenían los profesores al manifestar "...la química en 
muchos aspectos es muy abstracta: cuando vemos átomos, cuando vemos moléculas. Entonces poder mostrar los modelos que encontramos en internet me facilita las cosas en la medida en que ya no queda simplemente en contarles que parece que es así sino que les puedo mostrar los modelos" (E1) y "...hace más vivencial una materia, en el que ya no hay que imaginar, no tienen que estar imaginando las cosas que uno habla y que ellos a veces ni se las imaginan" (E6). Al respecto Resa (2017) muestra que las representaciones mediante animaciones son útiles y gustan a los estudiantes para la explicación de modelos relacionados con la química y el aprendizaje enriquecido por las TIC es más efectivo que los enfoques tradicionales de enseñanza (Tapasco y Giraldo, 2017).

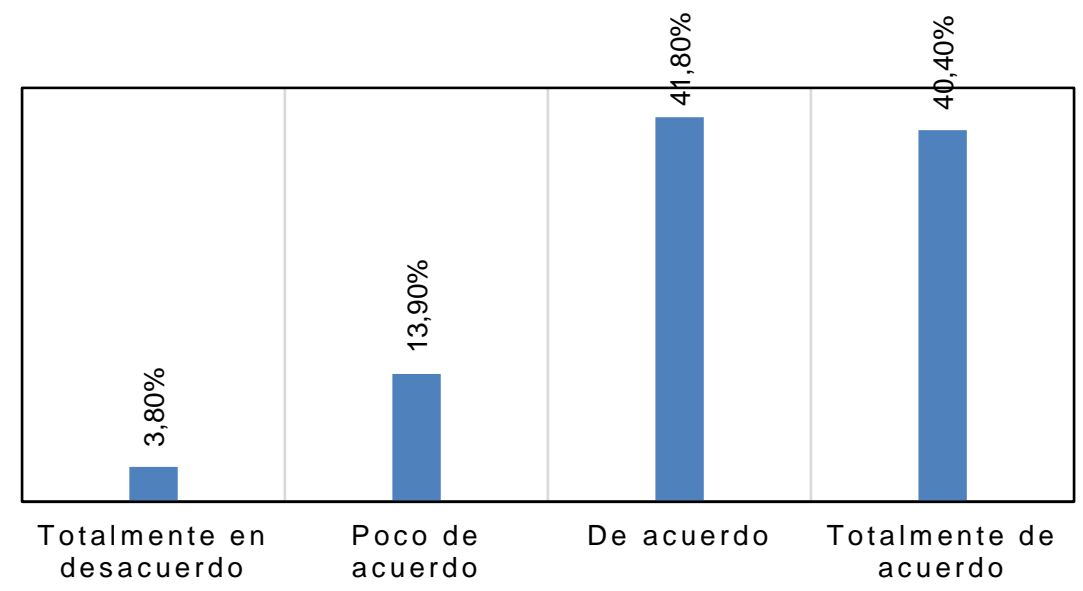

Fig. 1: Utilización de las TIC simplifica el aprendizaje

Sin embargo más de la mitad de los estudiantes $(54,4 \%)$ consideraron que no hay suficiente número de herramientas TIC en química para realizar una clase donde se puedan implementar (ver figura 2). Una opinión similar tenían (50\%) los profesores entrevistados (ver tabla 3).

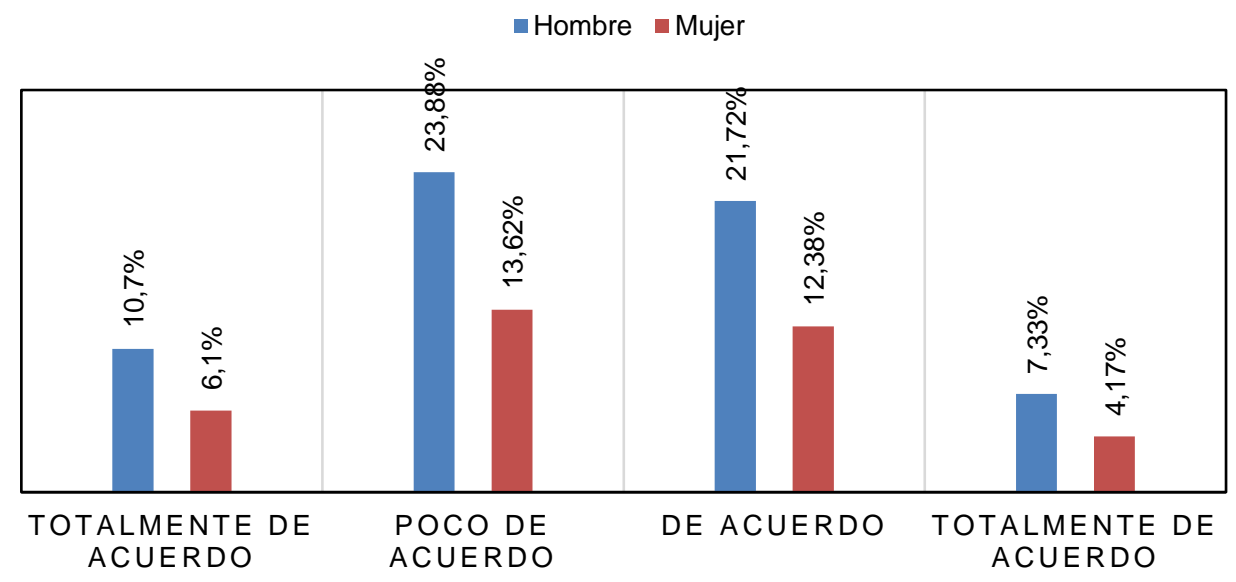

Fig. 2: Uso de las TIC en química por sexo

\section{Enseñanza de la química en el aula}

Con relación a las clases de química en general los docentes reconocieron que sus clases frecuentemente (4) y siempre (2) se centraban en una exposición donde el profesor transmitía unos conocimientos, mientras los alumnos escuchaban, tomaban notas y tenían la oportunidad de preguntar. Sin embargo, la mayoría de ellos opinaron que los esquemas de tablero y tiza, nunca, o en algunas ocasiones, son irremplazables (ver tabla 3). Una opinión similar tenían los estudiantes al respecto: para el 46,2\% de los estudiantes, sus profesores de química siempre preparaban sus clases de manera magistral (figura 3) y el $56 \%$ de los estudiantes encuestados no se mostraron muy de acuerdo, o estaban totalmente en desacuerdo, con que el tablero o pizarra y la tiza o marcador sean elementos insustituibles a la hora de una clase (figura 4).

La situación anterior fue corroborada repetidamente por los estudiantes: el $43,1 \%$ de los participantes estimaba que sólo algunas veces los profesores de química utilizaban las TIC en sus clases y para el $31,1 \%$ su uso era más frecuente, mientras que para el 16,3\% afirmaba que sus profesores nunca hacían uso de ellas (ver figura 5 y tabla 4). Ante estos resultados no es sorprendente encontrar docentes que declaren, 
refiriéndose al uso de las TIC en las clases de química: "pues la verdad es que en la química he trabajado muy poco porque los recursos que hay en la institución son mínimos" (E4) y que la solución que planteen algunos maestros sea "que el gobierno haga unos aportes para el mantenimiento porque esos aparatos son sumamente delicados y costosos" (E3).

Tabla 3: Concepciones de los docentes sobre la enseñanza de la química en el aula de clase

\begin{tabular}{|l|c|c|c|c|}
\hline \multicolumn{1}{|c|}{ Aseveraciones } & Nunca & $\begin{array}{c}\text { Algunas } \\
\text { Veces }\end{array}$ & $\begin{array}{c}\text { Frecuente- } \\
\text { mente }\end{array}$ & Siempre \\
\hline $\begin{array}{l}\text { 1. Prepara sus clases de manera magistral, centrando la mayor } \\
\text { parte del tiempo a su exposición mediante ejemplos y ejercicios; } \\
\text { mientras los estudiantes prestan atención, toman apuntes y } \\
\text { preguntan para resolver sus dudas. }\end{array}$ & & & & 2 \\
\hline 2. El tablero o pizarra y la tiza o marcador son irremplazables & 2 & 2 & 1 & 1 \\
\hline $\begin{array}{l}\text { 3. Considera que no hay suficientes herramientas de química } \\
\text { que permitan el uso de las TIC }\end{array}$ & 1 & 3 & 2 & \\
\hline 4. En su planeación anual incluye el uso y manejo de las TIC & & 2 & 2 & 2 \\
\hline
\end{tabular}

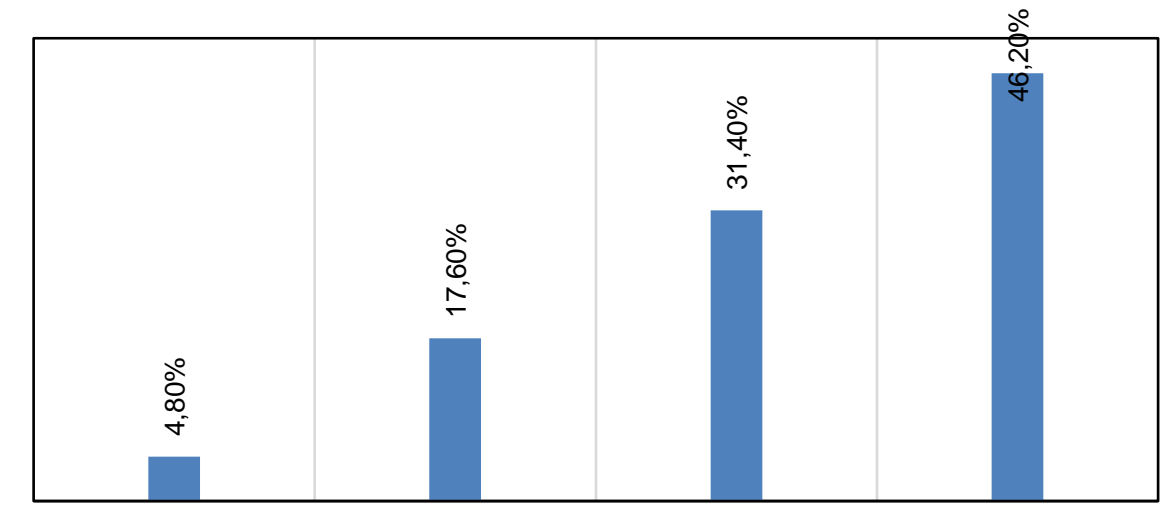

Nunca ALGunas VECESfRECuENTEMENTE SIEMPRE

Fig. 3: Las clases de química son magistrales

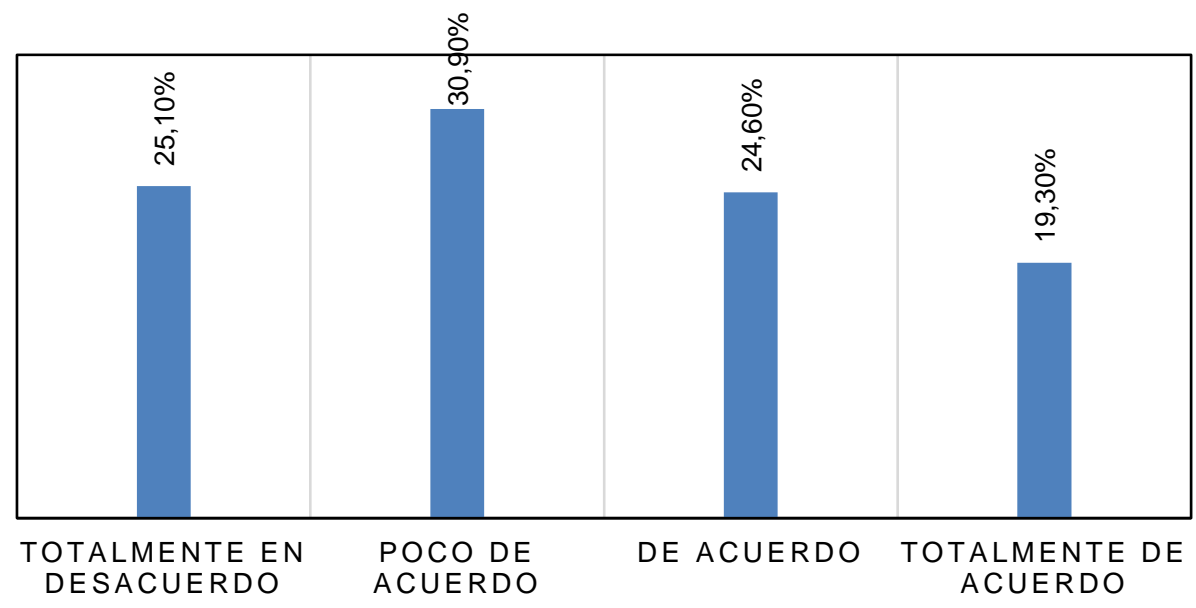

Fig. 4: El tablero y la tiza son indispensables

Otro aspecto de interés es la incorporación que los docentes hacen de las TIC en su vida profesional. Inicialmente se indagó sobre los recursos a los cuales recurrían cuando necesitaban realizar una consulta en la materia; las respuestas obtenidas mostraron que la primera fuente a la que acuden los profesores es internet, en segundo lugar libros o enciclopedias y en tercer lugar sus pares; con respecto a este último aspecto un profesor declaró: "....a través de la experiencia de otros profesores ahí mismo en el computador, poder mirar qué cosas más podría yo mejorar en el proceso” (E5). 


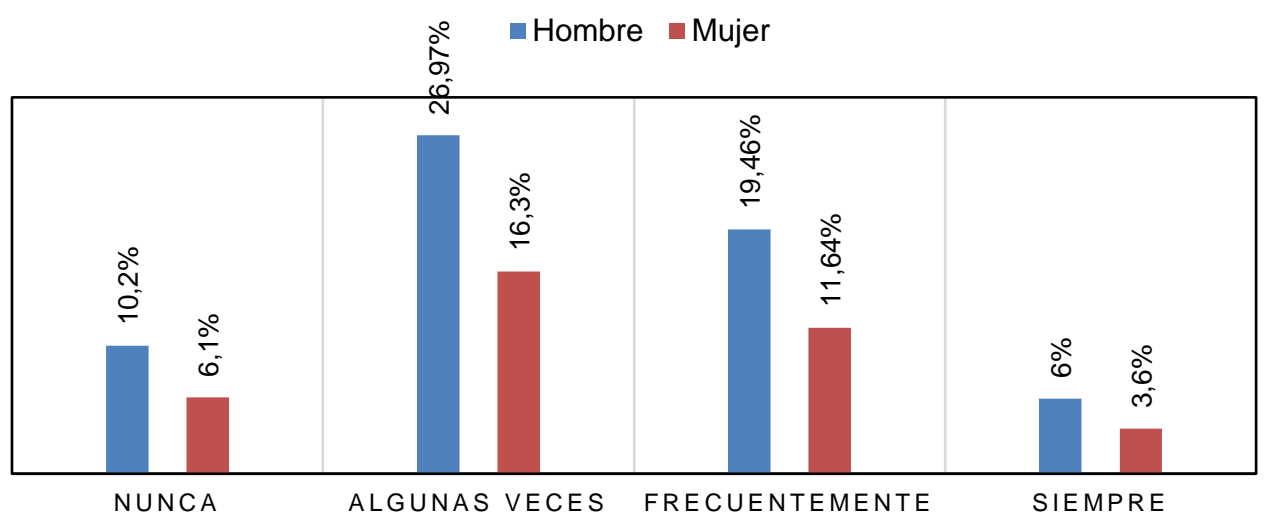

Fig. 5: Uso de las TIC por los profesores de química en clase

Tabla 4: Número de actividades que incorporan el uso de TIC en clase de química

\begin{tabular}{|c|c|c|c|}
\hline \multicolumn{2}{|c|}{ Número de actividades o clases que incorporan el uso de las TIC } & Frecuencia & Porcentaje \\
\hline 1. & Ninguna & 49 & $23,5 \%$ \\
\hline 2. & Entre 1 y 5 & 121 & $57,5 \%$ \\
\hline 3. & Entre 6 y 10 & 38 & $18 \%$ \\
\hline 4. & Más de 10 & 2 & $1 \%$ \\
\hline \multicolumn{3}{|c|}{210} \\
\hline
\end{tabular}

En la tabla 5 se presentan las frecuencias absolutas y relativas de los medios de consulta que utilizaban con mayor frecuencia los estudiantes cuando tenían que realizar una consulta sobre un tema de química; en ella se pone de manifiesto que, al igual que los profesores, a la primera fuente que recurrían es internet (98\%).

Tabla 5: Medios de consulta utilizadas por los estudiantes en la consulta de química

\begin{tabular}{|l|c|c|}
\hline \multicolumn{1}{|c|}{ Herramienta de consulta } & No. estudiantes & Porcentaje \\
\hline Internet & 206 & $98 \%$ \\
\hline Maestros & 165 & $79 \%$ \\
\hline Compañeros & 163 & $78 \%$ \\
\hline Libros y enciclopedias & 161 & $77 \%$ \\
\hline \multicolumn{2}{|c|}{210} \\
\hline
\end{tabular}

También es de mencionar el uso de trabajos asignados por los profesores a sus estudiantes usando TIC "...en clase se le dejan trabajos para que ellos los hagan en la casa (refiriéndose a trabajos usando TIC)" y "hay temas de consulta y hay temas para explicar en clase" (E4); "los estudiantes consultan, yo también consulto y confrontamos la información para fortalecer los procesos" (E5).

\section{Las TIC en el aula de química}

Las formas como más frecuentemente se incorporaron las TIC en el aula, durante las clases de química, correspondían al uso de videos y artículos, seguido de presentaciones y desarrollo de las clases usando Power Point: "en algunos momentos si utilizo videos... información para consulta," "la tarea es escribir ¿qué vio en el video?"; "si utilizo videos, utilizo a través de las diferentes páginas de internet, información para consulta". "Me gustan mucho los videos" (E5); "tengo videos, DVD, los busco y los quemo y eso se les pasa allí... se seleccionan los videos que sean buenos para la clase, según el tema y es interesante ver los videos" (E6); "Yo como le digo, están muy dedicados a los videos, está el manejo del video beam... traen videos (los estudiantes)" (E3). Incluso los mismos estudiantes para tener evidencias de sus trabajos graban las actividades que luego son enviadas a los respectivos profesores de química "ellos elaboran videos, los suben a internet" (E1). Es decir para el $37,5 \%$ de los estudiantes, sus profesores presentaban videos y artículos para fortalecer o enriquecer sus prácticas (Tabla 6). Al respecto un profesor declaró: "entonces yo insisto en la retroalimentación, constante retroalimentación. Se está hablando de un tema y de una vez se puede retroalimentar algo que se necesita ahí y que ya se vio clases atrás. Y lógicamente ver utilizando las TIC facilita más eso... claro, siempre se piensa en eso" (E6). 
Continuando con el análisis de los estudiantes, para el 26,9\% de ellos, el uso de videos en la clase de química tenía lugar sólo algunas veces. Este es un aspecto reiterativo y se evidencia una vez más en la tabla 6 . El $72,9 \%$ de los estudiantes reciben sugerencias de sus docentes para ver programas 0 documentales relacionados con la materia, mientras que un 40,5\% manifiestaron que algunas veces las clases de química son presentadas en Power Point u otra forma didáctica audiovisual.

Se deduce de lo anterior que la forma como los profesores integran las TIC continúa bajo el enfoque tradicional, centrada en el contenido y en el profesor y el alumnado siendo sujeto pasivo que recibe conocimientos (Marcelo, et al., 2016). En cambio, para aquellas actividades de aprendizaje que pudieran representar una enseñanza basada en el alumnado, el nivel de conocimiento era bajo o nulo ya que en ningún momento los profesores ni los estudiantes manifestaron por ejemplo incluir prácticas como animaciones a partir de esos videos, de tal forma que se ofrecieran oportunidades para pensar, cuestionar, construir y reconstruir los procesos que se deseaban animar (Hoban y Nielsen 2010, Hoban y Nielsen 2014).

Tabla 6: Apreciaciones por parte de los estudiantes con respecto a la incorporación de las TIC en las clases de química

\begin{tabular}{|c|c|c|c|c|}
\hline Aseveraciones & Nunca & $\begin{array}{c}\text { Algunas } \\
\text { veces }\end{array}$ & $\begin{array}{l}\text { Frecuente- } \\
\text { mente }\end{array}$ & Siempre \\
\hline $\begin{array}{l}\text { 1. El profesor(a) presenta videos y artículos de interés para } \\
\text { fortalecer sus clases de química }\end{array}$ & $17.3 \%$ & $26.9 \%$ & $37.5 \%$ & $18.3 \%$ \\
\hline $\begin{array}{l}\text { 2. El profesor(a) presenta o recomienda películas y/o } \\
\text { programas televisados relacionados con la química }\end{array}$ & $27.1 \%$ & $33.8 \%$ & $28.6 \%$ & $10.5 \%$ \\
\hline 3. Las clases son presentadas en medios audiovisuales & $28.6 \%$ & $40.5 \%$ & $22.9 \%$ & $8.1 \%$ \\
\hline 4. Se realizan laboratorios virtuales de química & $58.4 \%$ & $17.7 \%$ & $17.2 \%$ & $6.7 \%$ \\
\hline $\begin{array}{l}\text { 5. El profesor(a) utiliza tutoriales para fortalecer y probar los } \\
\text { conocimientos en química. }\end{array}$ & $44.3 \%$ & $30.5 \%$ & $19.5 \%$ & $5.7 \%$ \\
\hline 6. Utiliza varios software adaptados y pertinentes a la química & $55.3 \%$ & $27.9 \%$ & $13.0 \%$ & $3.8 \%$ \\
\hline 7. El Profesor(a) crea o actualiza un blog para química. & $55.5 \%$ & $22.0 \%$ & $15.8 \%$ & $6.7 \%$ \\
\hline $\begin{array}{l}\text { 8. El profesor(a) asigna tareas y actividades de química } \\
\text { haciendo uso del blog o página del colegio. }\end{array}$ & $62.7 \%$ & $14.4 \%$ & $17.2 \%$ & $5.7 \%$ \\
\hline $\begin{array}{l}\text { 9. El profesor registra en videos o fotos actividades de su clase } \\
\text { de química }\end{array}$ & $46.2 \%$ & $28.1 \%$ & $19.0 \%$ & $6.7 \%$ \\
\hline $\begin{array}{l}\text { 10. El registro de videos o fotos se publica en el blog de química, } \\
\text { plataforma o redes sociales }\end{array}$ & $71.2 \%$ & $15.4 \%$ & $9.1 \%$ & $4.3 \%$ \\
\hline $\begin{array}{l}\text { 11. El profesor evalúa haciendo uso de medios electrónicos: } \\
\text { tablero digital, teléfono móvil, correo electrónico u otro. }\end{array}$ & $65.2 \%$ & $16.9 \%$ & $13.5 \%$ & $4.3 \%$ \\
\hline $\begin{array}{l}\text { 12. El profesor resuelve dudas usando medios electrónicos: chat, } \\
\text { redes sociales, correo electrónico, etc. }\end{array}$ & $56.2 \%$ & $15.7 \%$ & $21.0 \%$ & $7.1 \%$ \\
\hline
\end{tabular}

Para más de la mitad $(58,4 \%)$ de los estudiantes nunca se habían desarrollado laboratorios virtuales y el $17,2 \%$ de los jóvenes algunas veces. Ante la escasez de recursos, no sorprende que los profesores de química vean limitadas las prácticas de laboratorio por no contar con los reactivos ni materiales de laboratorio que permitan desarrollarlas, privando a sus estudiantes de prácticas científicas a través de procesos de indagación donde el laboratorio es pertinente para promover competencias científicas (Högstrom, Ötander y Benckert, 2010). Aquí también las TIC pueden contribuir a subsanar esta problemática de una inmensa mayoría de las instituciones colombianas mediante laboratorios virtuales. De hecho algunos profesores no desconocen esta posibilidad que brindan las TIC, incluso aquellos que se declaran abiertamente indiferentes a ellas: "el estudiante visualice algunos elementos del laboratorio"; "...hemos visto laboratorios, hemos visto resúmenes, hemos avanzado" (E3); "...me gustan algunas simulaciones que hay de laboratorios" (E5); "acceden a internet a laboratorios virtuales" (E1).

Sin embargo el $44,3 \%$ negaron que sus docentes usaran tutoriales y simuladores para la enseñanza de la química, mientras que los que declararon que siempre o algunas veces los había usado fueron el 5,7\% y $30,5 \%$ respectivamente. Un comportamiento similar se observó en cuanto al uso de programas o software para química: más de la mitad de los estudiantes $(55,3 \%)$ respondieron que sus profesores nunca utilizaban programas especiales para la materia, un $27,9 \%$ afirmó que en algunas ocasiones y el $13 \%$ consideró una práctica frecuente la utilización de este recurso. Lo anterior concuerda con los datos suministrados por los profesores sobre la incorporación de TIC en sus clases (Tabla 7). 
Tabla 7: Utilización de las TIC por los docentes

\begin{tabular}{|c|c|c|c|c|}
\hline Aseveraciones & Nunca & $\begin{array}{l}\text { Algunas } \\
\text { Veces }\end{array}$ & $\begin{array}{l}\text { Frecuente- } \\
\text { mente }\end{array}$ & Siempre \\
\hline 1. Reviso diariamente mi cuenta o cuentas de correo electrónico & 1 & 2 & & 3 \\
\hline 2. Crea un blog para química o actualiza el que tiene & 4 & & & 2 \\
\hline $\begin{array}{l}\text { 3. Asigna tareas y actividades de química haciendo uso del blog o el } \\
\text { correo electrónico }\end{array}$ & 3 & 1 & 1 & 1 \\
\hline $\begin{array}{l}\text { 4. Revisa en la red videos y artículos de interés para fortalecer sus } \\
\text { clases de química }\end{array}$ & 1 & & 3 & 2 \\
\hline 5. Realiza sus propios videos y registros. & 5 & & & 1 \\
\hline $\begin{array}{l}\text { 6. Sus registros y videos son publicados en el blog de química o en la } \\
\text { página del colegio }\end{array}$ & 5 & & & 1 \\
\hline $\begin{array}{l}\text { 7. Sus clases de química son presentadas en Power Point o haciendo } \\
\text { uso de cualquier otro medio audiovisual }\end{array}$ & 1 & 4 & 1 & \\
\hline 8. Evalúa haciendo uso del móvil, correo electrónico, tablero digital, etc. & 2 & 4 & & \\
\hline 9. Han creado una wiki para química & 6 & & & \\
\hline 10. Realiza laboratorios virtuales de química con sus estudiantes & 4 & 1 & 1 & \\
\hline $\begin{array}{l}\text { 11. Fortalece y pone a prueba el conocimiento de sus estudiantes en } \\
\text { química mediante el uso de tutoriales }\end{array}$ & 5 & 1 & & \\
\hline 12. Utiliza varios software adaptados y pertinentes a la química & 2 & 4 & & \\
\hline
\end{tabular}

Con respecto a los programas de química que son seleccionados por los docentes a la hora de desarrollar los temas de su respectiva área, se les entregó una lista de 22 programas para que marcaran la regularidad con la que eran utilizados. La lista de programas de química mostrada a los maestros corresponde, por un lado, a dos programas para simular laboratorios (VLabQ y MovieLab) que el Gobierno Nacional de Colombia ha puesto a disposición de los profesores y estudiantes, el primero se encuentra gratis en la web a través de los portales "colombiaaprende" y "eduteka", y el segundo es donado por el gobierno municipal a los colegios de estratos sociales inferiores; y por otro lado a veinte programas que en consideración de de Pedro Fernández Cortés (responsable del Programa de Tele-formación de la Coruña-España) son los mejores para la docencia en la enseñanza de la química a nivel de ESO y bachillerato (Ver Tabla 8).

Tabla 8: Programas para el área de química disponible en internet

\begin{tabular}{|l|l|}
\hline \multicolumn{2}{|c|}{ Programas para la enseñanza de la química en el nivel de bachillerato } \\
\hline VLabQ & Prolkon \\
\hline MoviLab & ChemLab \\
\hline WebLab Viewer Pro & GlassyChemistry \\
\hline Rasmol & Ptoe \\
\hline Bondit & Integral Scientist Periodic Table \\
\hline Chemsketch & Perlib \\
\hline IsisDraw & Atoms, Bonding and Structure \\
\hline Molecular Weight Calculator & Atoms, simbols and Equation \\
\hline Chemical Calculator & Q- Geum \\
\hline Chembalanze Wizard & Chemistry Tutor \\
\hline Stoichiometry Studio & Turbocalc \\
\hline Otro: ¿cuál? & \\
\hline
\end{tabular}

Los seis profesores dejaron en blanco esta tabla, de tal forma que los resultados obtenidos permitieron determinar que los docentes, o desconocen, o no utilizan estas herramientas para enriquecer los procesos de enseñanza-aprendizaje en el área de química. Aún más, ni siquiera tenían conocimiento de aquellos programas promovidos por el Ministerio de Educación Nacional. De otro lado se esperaría que el número de profesores que tienen blogs para guiar sus actividades vaya en aumento: "...hoy día tenemos unos blogs" (E1); "el año pasado 
trabajaba (refiriéndose al uso de las TIC) y tengo un blog" (E4). No obstante, para el 55,5\% de los estudiantes sus profesores de química no tienen un blog para la materia, un $22 \%$ opina que algunas veces sus profesores crean uno y aún en el caso de aquellos que lo tienen, el 62,7\% de los estudiantes declaró que nunca les habían asignado actividades curriculares a través de él o de la página del colegio. Además se puede observar que el $46,2 \%$ de los estudiantes respondieron que sus profesores nunca hacían un registro audiovisual de las actividades que se realizaban en la materia, el $28,1 \%$ que tan sólo algunas veces.

La encuesta aplicada a los profesores y la posterior entrevista reveló que las temáticas en las que los profesores han incorporado las TIC en la enseñanza de la química eran: gases, propiedades de la materia, reacciones químicas, enlaces, polímero, petróleo, caucho y átomo de carbono. Los docentes realizaron los siguientes comentarios, entre otros: Enlaces químicos: “...le ayudan a hacer más entendible a los estudiantes los temas que tienen por lo menos de enlaces, explicación que lo haga uno magistralmente, ¿cómo se hace la unión entre enlaces?" (E4); Química y el desarrollo tecnológico: "el avance en cuanto al plástico que es lo máximo que ha habido de revolución" (E3); "en eso de polímeros también estoy utilizando demasiado la sala de audiovisuales para eso" (E3); "la preparación de pólvora por ejemplo en video y lo vemos" (E3); Reacciones químicas: "...bueno, todo ese tema de reacciones químicas también" (E4); Estructura y modelación química: "...química orgánica... yo traigo ya las estructuras montadas" y "les puedo mostrar los modelos" (E1); tabla periódica y ley periódica: "está la tabla periódica y para aprenderse los símbolos, en el blog mío tengo la tabla periódica cómo está, cómo se indica la posición, está explicado cómo son los elementos, cuáles son los elementos metales e identificarlos... no metales... la clasificación, cómo está ordenada, cómo está ordenada, la tabla periódica" (E4).

La encuesta a los estudiantes evidenció que para el $65,2 \%$ los medios electrónicos como teléfono móvil, correo electrónico, tablero digital, etc. no eran alternativas para ser evaluados por sus docentes. El 56,2\% afirmaron que el profesor de química no interactúaba con ellos por medio de herramientas electrónicas como el chat, las redes sociales y el correo como medios para exponer sus dudas y/o recibir respuestas (Tabla 6).

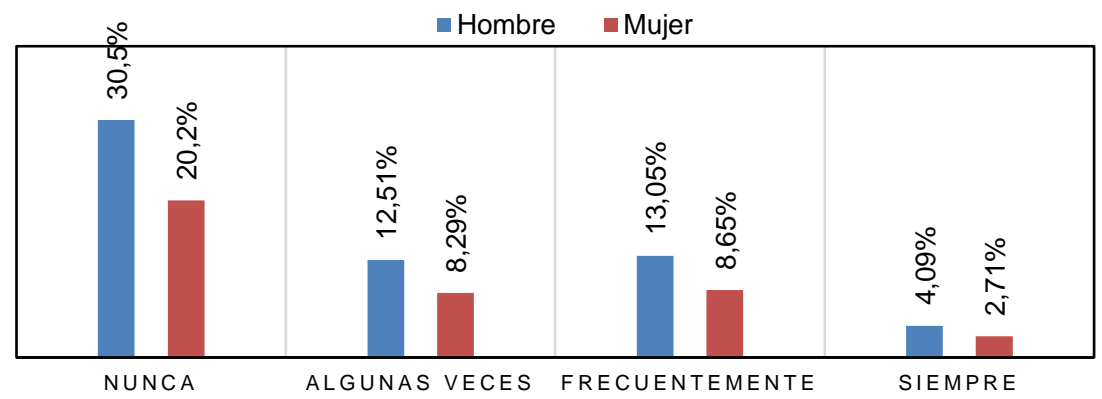

Fig. 6: Utiliza las redes sociales con fines educativos en el área de química

Un comportamiento similar se observa en la figura 6 donde se muestra el uso de las redes sociales con fines educativos: el $50,7 \%$ de los estudiantes declararon no haberlas usado nunca, mientras que el $21,7 \%$ declararon haberlas usado frecuentemente. De otro lado, se advierte que para casi las $3 / 4$ partes de los participantes nunca había creado un wiki para el área de química (ver figura 7).

En este estudio se ha puesto de manifiesto que en ningún caso los datos suministrados por los estudiantes fueron incoherentes por los suministrados por sus profesores. . El estudio ha mostrado también que hay un limitado uso de las TIC en la enseñanza de la química y que de ser empleadas adoptan una orientación donde el alumno es un sujeto pasivo que recibe información a través de ellas, lo cual coincide con el estudio de otros autores (Marcelo, et al., 2016), y demuestra carencias de empoderamiento digital por parte de los profesores de química (Jiménez-Pitre, et al., 2017).

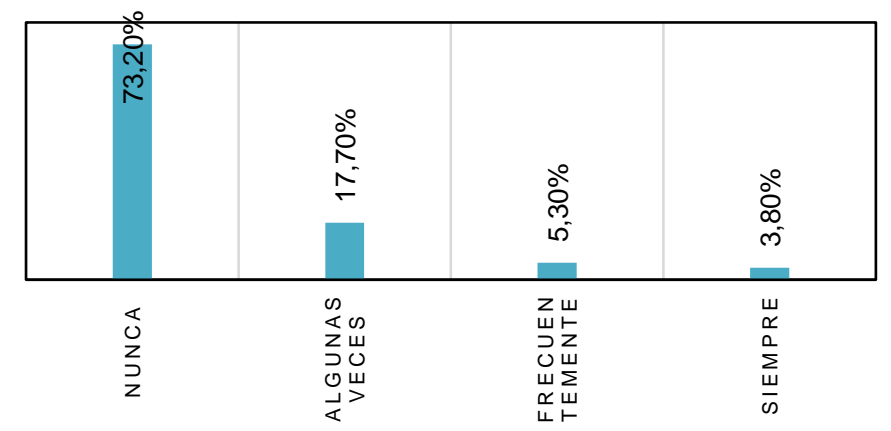

Fig. 7: Los estudiantes han creado un wiki para el área de química 


\section{CONCLUSIONES}

De acuerdo a los resultados obtenidos se puede concluir que: 1) los profesores de química en su mayoría preparan sus clases de manera magistral: haciendo exposición del tema, realizando ejemplos, ejercicios y resolviendo dudas; ocasionalmente se incluye algun método interactivo que incluya el uso de las TIC: como diapositivas, presentación en Power Point o video. 2) Las TIC son un apoyo pedagógico en la enseñanza de la química, sin embargo los profesores desconocen o no hacen uso de ellas: ninguno ha manejado programas, tutoriales o simuladores para química, ninguno hace uso de las redes sociales o plataformas con fines educativos; no han creado un blog para química y los que lo tienen no lo actualizan, tampoco recurren a esta opción, o a la página de la institución para asignarles actividades. 3) Los medios electrónicos como correo, tablero digital, etc. no son alternativas frecuentes que usen sus profesores para evaluarlos ni resolver dudas. 4) Tanto profesores como estudiantes recurren a internet como primera opción para hacer una consulta. 5) Los temas en los que más frecuentemente se incorporan las TIC a la enseñanza de la química son: gases, propiedades de la materia, reacciones químicas, tabla periódica, enlaces, átomo de carbono, equilibrio, polímeros, petróleo y caucho.

\section{REFERENCIAS}

Albion, P. R., Jamieson-Proctor, R., y Finger, G., Auditing the TPACK Confidence of Australian Pre-Service Teachers: The TPACK Confidence Survey (TCS), Paper presented at the 21st International Conference of the Society for Information Technology y Teacher Education, San Diego, U.S.A., march 19 (2010)

Barroso, J. y Llorente, M.C., La evaluación en teleformación y las herramientas para la creación de exámenes para la red, Posibilidades de la teleformación en teleformación en el espacio europeo de educación superior, Granada, Octaedro Andalucía, 247-268 (2007)

Bernal, H., "Radio Sutatenza" De la Realidad a la Utopía, 5-73, Acción Cultural Popular, Bogotá, Colombia (2005)

Cabero, J., Las TICs en la enseñanza de la química: aportaciones desde la Tecnología Educativa. Química, vida y progreso, Murcia: Asociación de químicos de Murcia, 1-34 (2007)

Cabero, J., Más allá de la planificación en la “Educación en Medios de Comunicación”, Comunicar, 8, $39-48$ (1997)

Cañal, P., El desarrollo de la competencia científica demanda y produce actitudes positivas hacia la ciencia y el conocimiento científico, en 11 ideas clave. El desarrollo de la competencia científica, $1^{\text {a }}$ Ed., Editorial GRAÓ, pp. 197216, Barcelona, España (2012)

Computadores para Educar. A más de un millón de niños les llegó tecnología. Bogotá D.C. Colombia (En línea) https://goo.gl/DJDoBi. Acceso octubre 2016 (2011)

Espuny, C., Gisbert, M., González, J., y Coiduras, J. Los seminarios TAC. Un reto de formación para asegurar la dinamización de las TAC en las escuelas, Edutec: Revistas electrónica de tecnología educativa, 34, 1-20, (En línea) https://goo.gl/Dr5YYo. Acceso: mayo 2016 (2010)

Hechter, R.P., Phyfe, L. D., y Vermette, L. A., Integrating Technology in Education: Moving the TPCK Framework towards Practical Applications, Education Research and Perspectives, 39(1), 136-152 (2012)

Hernandez, R., Baptista L.P., Fernández C., Metodología de la investigación, McGraw Hill, México, (2010)

Hinojo, F.J. y Fernández, F., Diseño de escala de actitudes para la formación del profesorado en tecnologías. Comunicar. Revista científica de comunicación y educación, 19, 120-125. https://goo.gl/4DwEcP. Acceso noviembre 2016 (2002)

Hoban, G., y Nielsen, W., The 5 Rs: A new teaching approach to encourage slowmations (student generated animations) of science concepts, Teaching Science, 56 (3), 33-38 (2010)

Hoban, G., y Nielsen, W., Creating a narrated stop-motion animation to explain science: The affordances of "Slowmation" for generating discussion, Teaching and Teacher Education 42, 68-78, (2014)

Högstrom, P., Ottander, C. y Benckert, S., Lab work and learning in secondary school chemistry: the importance of teacher and student interaction, doi: 10.1007/s11165-009-9131-3, Research in Science Education, 40, 505-523 (2010)

Jiménez-Pitre, I. A, Martelo, J.R. y James J. D. C, Escuela de gobierno basada en TIC: Determinante para la Accesibilidad e Integralidad del Empoderamiento Digital, Información Tecnológica, 28(5), 75-86, (En línea) doi: 10.4067/S0718-07642017000500010, Acceso: diciembre 2017 (2017)

Marcelo, C., Yot, C., y Perera, V.H., El conocimiento tecnológico y tecnopedagógico en la enseñanza de las ciencias en la universidad. Un estudio descriptivo, Enseñanza de las Ciencias, 34(2), 67-86 (2016)

Martínez, L.D. y Quijano, M. H., Propuesta didáctica para la enseñanza y el aprendizaje de la tabla periódica desde una perspectiva histórica y epistemológica, Il Congreso Nacional de investigación en educación en ciencias y tecnología, 115-126, Cali, Colombia 21 al 23 de junio, (2010)

MINTIC. Ministerio de las Tecnologías y Comunicaciones, Colombia gana premio mundial por su modelo de acceso a las TIC y al conocimiento (En línea) https://goo.gl/S72dGc. Acceso: 16 de mayo de 2012, Bogotá D.C. Colombia (2012) 
Morales, P., Investigación experimental, diseños y contrastes de medias. Universidad Pontifícia Comillas, Madrid, España (2013)

Niess, M.L., Preparing teachers to teach science and mathematics with technology: Developing a technology pedagogical content knowledge, Teaching and Teacher Education, 21(5), 509- 523, (En línea) https://doi.org/10.1016/j.tate.2005.03.006. Acceso: octubre 2012 (2005)

OCDE, Organización para la cooperación y el desarrollo económico, PISA 2015: Resultados Clave, OCDE (En Línea) https://goo.gl/ZE9i4t, acceso: Octubre 2016 (2016)

Orlik, Y., Química: métodos de enseñanza y aprendizaje, Grupo Editorial Iberoamericano, Méxido. D.F. (2002)

Oviedo, H., y Campo, Arias, A., Aproximación al uso del coeficiente alfa de Cronbach, Revista Colombiana de Psiquiatría, 34(4), 572-580 (En línea) https://goo.gl/SNSCJ5, Acceso: septiembre 2016 (2005)

Presidencia de la República, Bases del Plan Nacional de Desarrollo 2014-2018, Todos por un Nuevo País, Paz, Equidad, Educación. Bogotá D.C. Gobierno de la República (2014)

Resa, K., Learning from contrasting molecular animations with a metacognitive monitor activity, Educación Química, 28, 181-194 (2017)

Salcedo, et. al., Tecnologías de la información y la comunicación en educación en Química, Universidad Pedagógica Nacional, Fondo Editorial Luis Eduardo Vásquez Salamanca, Bogotá, Colombia (2008)

Sancho, Gil, J. M., De TIC a TAC, el difícil tránsito de una vocal, Investigación en la Escuela, 64, 19-30. https://goo.gl/2n1b5n, Acceso en febrero 2016, (2008)

Shulman, L., Those who understand: Knowlwdge growth in teaching, Educational Researcher, 15, 4-14 (1986)

Talanquer, V., ¿Qué conocimientos distinguen a los buenos maestros de química?, Educación química, 15 (1), 60-66, (En línea) https://goo.gl/Z9vo73, acceso octubre 2016 (2004)

Tapasco, O. A. y Giraldo, J. A., Estudio comparativo sobre Percepción y uso de las TIC entre Profesores de Universidades Públicas y Privadas, Formación Universitaria, 10(2), 3-12 (En línea) doi: 10.4067/S071850062017000200002 , acceso diciembre 2017 (2017) 